\title{
Data-Driven Evaluation for Demand Flexibility of Segmented Electric Vehicle Chargers in the Korean Residential Sector
}

\author{
Keon Baek ${ }^{1}{ }^{\mathbb{D}}$, Sehyun $\mathrm{Kim}^{2}{ }^{2}$, Eunjung Lee ${ }^{1}$, Yongjun $\mathrm{Cho}^{1}$ and Jinho Kim ${ }^{1, *}$ \\ 1 Gwangju Institute of Science and Technology, School of Integrated Technology, Gwangju 61005, Korea; \\ keonbaek@gm.gist.ac.kr (K.B.); jk151149@gist.ac.kr (E.L.); dragonjun3107@gm.gist.ac.kr (Y.C.) \\ 2 Korea Electric Power Corporation, Naju 58322, Jeollanam-do, Korea; alexsehyunkim@gmail.com \\ * Correspondence: jeikim@gist.ac.kr; Tel.: +82-62-715-5322
}

check for updates

Citation: Baek, K.; Kim, S.; Lee, E.; Cho, Y.; Kim, J. Data-Driven

Evaluation for Demand Flexibility of Segmented Electric Vehicle Chargers in the Korean Residential Sector. Energies 2021, 14, 866. https://doi. org/10.3390/en14040866

Academic Editors: Tek Tjing Lie and Victor Becerra

Received: 20 December 2020

Accepted: 5 February 2021

Published: 7 February 2021

Publisher's Note: MDPI stays neutral with regard to jurisdictional claims in published maps and institutional affiliations.

Copyright: (c) 2021 by the authors. Licensee MDPI, Basel, Switzerland. This article is an open access article distributed under the terms and conditions of the Creative Commons Attribution (CC BY) license (https:/ / creativecommons.org/licenses/by/ $4.0 /)$.

\begin{abstract}
The rapid spread of renewable energy resources has increased need for demand flexibility as one of the solutions to power system imbalance. However, to properly estimate the demand flexibility, demand characteristics must be analyzed first and the corresponding flexibility measures must be validated. Thus, in this study, a novel approach is proposed to evaluate the demand flexibility provided by Electric Vehicle Chargers (EVC) in the residential sector based upon a new process of electric charging demand characteristic data analysis. The proposed model estimates the frequency, consistency, and operation scores of the flexible demand resource (FDR) during identified ramp-up/down intervals presented in our previous work. The scores are included in the components that calculate the flexibility score referring that the closer it is to 1, the higher utilization as an FDR. A case study was conducted by considering EV user segmentation based on their demand characteristic analysis. The results confirm that flexibility scores of segmented EVC groups are about 0.0273 in ramp-up and ramp-down intervals. Based on the experimental results, the flexibility score can be utilized for multi-dimensional analysis and verification in perspectives of seasonality, participation time interval, customer group classification, and evaluation. Thus, the proposed method can be used as an indicator to determine how a segmented EVC group is adequate to participate as an FDR while suggesting meaningful implications through EVC demand data analysis.
\end{abstract}

Keywords: demand response; demand flexibility; electric vehicle; data analysis

\section{Introduction}

Renewable energy resources have been replacing fossil fuels under the increasing pressure for clean and safe energy, and research on distributed energy resources (DERs) of microgrids is underway to implement so-called decarbonization policies. Unlike these purposes, renewable energies such as solar and wind power are characterized by volatility in power generation. Since the expansion of renewable energy in the distribution network causes mismatch between power supply and demand balance and weakens economics of power system operation, the scale of the ancillary service market inevitably increases. In addition, while the global energy demand continues to grow every year, the expansion of power generation and transmission infrastructure is not keeping this pace. To overcome demand spikes that only take place for a few hours of the one-year period, the grid network must be over-designed and approximately $20 \%$ of the rated capacity of generators participating in the energy market should be typically reserved [1,2]. Under certain situations of energy imbalance, the flexibility of the network and resources is becoming an important issue to ensure the stability of the power system.

The term flexibility has long been mentioned in schools or institutions, and its meaning has changed over time reflecting the problem faced by the system [3]. Under the environment of utility privatization and competition since 1990s, system operators have used the term to define power resources that endure rapid changes in generation price [4]. In addition, the meaning has been expanded to identify generation and demand resources 
for the purpose of supplementing the intermittent power generation due to the spread of renewable energy [5].

The main feature of flexible generation resources can be the rapid ramp up and down rate characteristics, and they should be able to operate efficiently at a lower minimum level throughout periods of high renewable energy output [6]. To quantify the supply characteristics of the generation resources, their flexibilities are measured by the indicators, namely magnitude, ramp frequency and response time for analyzing the power system flexibility according to the different temporal and spatial scales [7].

In terms of demand resources, to achieve the economic balance of supply and demand against irregular energy production, demand side management (DSM) is being developed vigorously. As a part of DSM mechanism, demand response (DR), in which utilities strategically (technically or through incentives) control the loads of participants to respond to grid network imbalances, can increase the flexibility of the power system in a costeffective manner. For example, flexible energy buildings that have applied a DR program using a smart carbon heating system or energy storage system (ESS) in building energy operations can shift the peak electricity consumption period to an off-peak period [8]. In these contexts, it is necessary to establish the resource flexibility from the viewpoint of demand sides.

The types of demand resources that can contribute to the system are largely classified into industrial, commercial, and residential loads. In particular, with the emergence of electric vehicles (EVs) along with a rapidly increasing residential demand nowadays, the possibility of utilizing EVs as a demand flexibility resource is being investigated. There are three types of EVs, classified by the degree that electricity is used as their energy source: battery electric vehicles (BEVs), plug-in hybrid electric vehicles (PHEVs), and hybrid electric vehicles (HEVs). Due to the advanced development of lithium-ion battery and charging infrastructure, there is a large potential for vehicle-to-grid (V2G) services [9]. For the step of V2G utilization, one-directional flow of power from the grid to vehicles (V1G) is currently being rolled out across California and in many places where EVs have become popular. V1G programs in California include Power Your Drive from San Diego Gas and Electric, EV-specific retail tariffs from Southern California Edison and a smart charging pilot program, namely Charge Forward, from pacific Gas and Electric in collaboration with Bayerische Motoren Werke [10].

Despite the current need for data analysis on the charging characteristics of EVs to make them as demand resources, it is quite difficult to track the usage history data for each EV individually, and there is a problem of privacy security invasion. Therefore, it is reasonable to consider resource evaluation based on the data of the EV charger (EVC) in which personal information is managed with encryption. Based on EVCs installed in certain places, a clear analysis of the load characteristics (e.g., periodicity, seasonality) must precede to use them as demand resources for securing flexibility.

A few related studies evaluated the general ability of EV fleet for power system operation, collected or surveyed large-scale EV charging data through demonstration projects, and analyzed the charging duration and amount of EVs [11-14]. However, to the knowledge of the authors, there are no reported studies evaluating the flexibility of EVCs with a valid formula that reflects EVC-data-driven characteristics, especially in a typical demand side resource perspective for grid network security. Therefore, there is a need to develop the method to estimate and evaluate the flexibility of EVC loads based on the data analysis results of EVC charging characteristics.

In the view of above, the main objectives and contributions of this study can be summarized as follows:

- A novel method to evaluate demand flexibility of the EVC based on guaranteed DR potential estimation is proposed.

- A new data analysis framework of EVC charging demand data to determine flexibility evaluation is suggested. 
The rest of the paper is organized as follows. In Section 2, a flexibility score estimation method for EVC flexibility evaluation is proposed and subsequently associated mathematical formulation is presented. This is followed by a description of EVC data analysis in Section 3. In Section 4, the results are presented accordingly to demonstrate and interpret the effectiveness of the proposed model. The conclusions are drawn in Section 5.

\section{Flexibility Score Estimation}

\subsection{Determination of the Ramp-Up/Down Intervals}

Before presenting a methodology of evaluating demand flexibility for EVC demand resources, it is necessary to define the ramp-up/down intervals for the flexible operation of DR. In the field of power systems, the term ramp-up/down comes from the capability to increase/decrease power generation in unit time, respectively [15]. In this study, the criteria for ramp-up/down intervals to mitigate the grid congestion due to sharp PV power reduction are self-referred from [16] (pp. 3). To mitigate fluctuations in net demand, flexible demand resources (FDRs) must be properly engaged (i.e., consuming or reducing more load if net demand sharply drops or increases, respectively). The start/end times of the ramp-up/down intervals were defined based on the minimum/maximum inclination of the net demand curves. The intervals for summer and winter, which are based on net demand in Jeju Island, Korea, are shown in Figure 1, and they are accordingly estimated as follows:

- $\quad$ Ramp-up interval: 12:00-17:59 (summer), 12:00-17:59 (winter).

- Ramp-down interval: 18:00-21:00 (summer), 18:00-20:00 (winter).

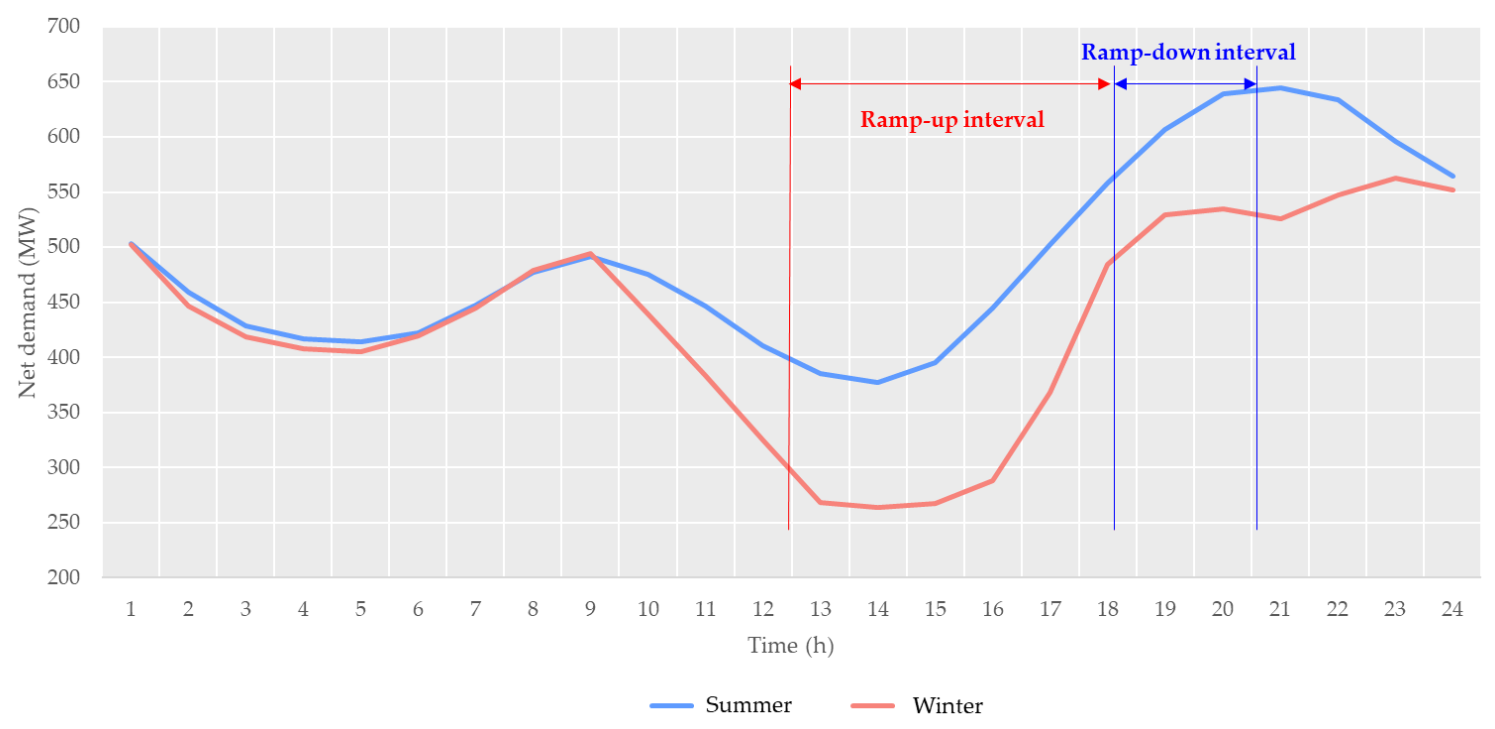

Figure 1. Ramp-up/down intervals based on the net-demand profile of summer and winter [15].

\subsection{Flexibility Score Formulation}

The demand flexibility of EVC is greatly affected by the EVC's rated capacity. However, the chargers in residential sectors have a $7 \mathrm{~kW}$ of common rated capacity with a lowvoltage, which is very small to be used individually. Therefore, it is essential to estimate the flexibility in the case of integrated EVC operation rather individual one. This paper designed a scoring method to estimate the flexibility of a clustered demand operation system based on the charging data characteristics of individual EVs. The formula evaluates separately according to the ramp-up and ramp-down time interval, respectively. Three indicators, namely frequency score $\left(F S_{k}\right)$, consistency score $\left(C S_{k}\right)$, and operation score $\left(O S_{k}\right)$, were formulated to quantify the availability of resources [16]. The attributes were scored between 0 and 1 , and the closer scores are to 1 , the more likely it is to be worth as a FDR. 
$F S_{k}$ indicates how often the demands are operated during the ramp-up and rampdown intervals. It involves to the utilization probability of the FDR during the periods of system demand fluctuation, which can be a major factor in estimating the flexibility via data. In addition, from the perspective of EVC aggregation operators, resources can be operated flexibly only when the EVs are charged at an appropriate ratio to the total demand capacity. Accordingly, $F S_{k}$ was designed as the ratio of operating EVCs to the entire segmented customer group over the whole period $(D)$, as shown in Equation (1). Based on the threshold load $\left(p^{\text {th }}\right)$, the operational state of the EVC $\left(u_{i, k, d}\right)$ was determined discretely as formulated in Equation (2):

$$
\begin{aligned}
& F S_{k}=\frac{1}{N_{D}} \cdot \frac{1}{N_{k}} \cdot \sum_{d \in D} \sum_{i \in I_{k}} u_{i, k, d} \\
& u_{i, k, d}=\left\{\begin{array}{cc}
1 & p_{i, k, d, t}>p^{t h} \\
0 & \text { otherwise }
\end{array}\right.
\end{aligned}
$$

$C S_{k}$ indicates the similarities between the estimated demand patterns and actual power consumption amounts. The FDRs with consistent power consumption could be determined to have the flexibility to perform well on stable capacity in response to the contractual participation offers. $C S_{k}$ was derived as shown in Equation (3) based on the root mean square percentage error $\left(R M S P_{k}\right)$ between min-max normalized actual demand $\left(p_{i, k, d, t}^{n}\right)$ and averaged demand pattern $\left(p_{k, t}^{n, a v g}\right)$. as in Equation (4). Min-max normalization of demand data was performed over the entire periods $(D)$ and groups $\left(I_{k}\right)$ as shown in Equation (5) and averaging normalized demand pattern was derived as formulated in Equation (6).

$$
\begin{gathered}
C S_{k}=1-\operatorname{RMSP}_{k} \\
\operatorname{RMSP}_{k}=\sqrt{\frac{1}{N_{D}} \cdot \frac{1}{N_{T}} \cdot \frac{1}{N_{k}} \cdot \sum_{d \in D} \sum_{t \in T} \sum_{i \in I_{k}}\left(\frac{p_{k, t}^{n, a v g}-p_{i, k, d, t}^{n}}{p_{i, k, t}^{n, a v g}}\right)^{2}} \\
p_{i, k, d, t}^{n}=\frac{p_{i, k, d, t}-\min _{t \in T, d \in D}\left(p_{i, k, d, t}\right)}{\max _{d \in D, t \in T}\left(p_{i, k, d, t}\right)-\min _{d \in D, t \in T}\left(p_{i, k, d, t}\right)} \\
p_{k, t}^{n, a v g}=\frac{1}{N_{D}} \cdot \frac{1}{N_{k}} \cdot \sum_{d \in D} \sum_{i \in I_{k}} p_{i, k, d, t}^{n}
\end{gathered}
$$

$O S_{k}$ evaluates the valid demand management volume of the FDR during ramp-up and ramp-down intervals. As formulated in Equation (7), $O S_{k}$ was calculated based on the actual charging demand of EVCs compared to the maximum peak power consumption for charging the entire EVs during the intervals. During the ramp-down interval, the more EVC being charged in the group, the greater the flexibility for reduction exists. On the other hand, during the ramp-up interval, the less EVC being charged in the group, the greater the flexibility increases through encouraging EV charging.

$O S_{k}=\left\{\begin{array}{c}1-\frac{1}{N_{D} \cdot \sum_{i \in N_{k}} \max _{t \in T}\left(p_{i, k, d, t}\right)} \cdot \sum_{d \in D} \sum_{t \in T} \sum_{i \in I_{k}} u_{i, k, d} \cdot p_{i, k, d, t} \text { When ramping up DR issued } \\ \frac{1}{N_{D} \cdot \sum_{i \in N_{k}} \max _{t \in T}\left(p_{i, k, d, t}\right)} \cdot \sum_{d \in D} \sum_{t \in T} \sum_{i \in I_{k}} u_{i, k, d} \cdot p_{i, k, d, t} \text { When ramping down DR issued }\end{array}\right.$

Finally, the flexibility score $\left(S_{k}\right)$ is defined by the relational expressions of the aforementioned attributes of $F S_{k}, C S_{k}$, and $O S_{k}$ as given in Equation (8).

$$
S_{k}=F S_{k} \cdot S C_{k} \cdot O S_{k}
$$




\section{Data Analysis of EV Chargers}

\subsection{Description of EV Charger Dataset}

This paper utilized the charging data of individual EVCs in a whole residential sector in Korea to estimate the flexibility in participating EVs for DR. The data was obtained from Korea Electric Power Corporation (KEPCO), a Korean monopolistic utility, which is in the business of supplying and operating EVCs. The charging data of $5148 \mathrm{EV}$ users were measured at 15-min intervals for a total of three months from 1 December 2019 to 29 February 2020. The initial data pre-processing was carried out to eliminate and impute data of EV customers with high missing rates and outliers (e.g., power consumption data exceeding the rate capacity of the charger). As a result, data of $4165 \mathrm{EV}$ users were finally able to analysis with the missing rate reduced from $7.68 \%$ to $0.78 \%$.

\subsection{Results of Data Analysis}

\subsubsection{Periodicity}

Customers with similar charging characteristics should be clustered to determine the flexibility level of the group. However, due to the prior necessity for identifying the characteristics of charging data, a periodicity analysis of the data was performed. The analysis result was confirmed through the 15-minutely and daily average charging amount within the measurement period as shown in Figure 2. There was a daily periodicity, and the average charging amount was lower on weekends and holidays than on weekdays.

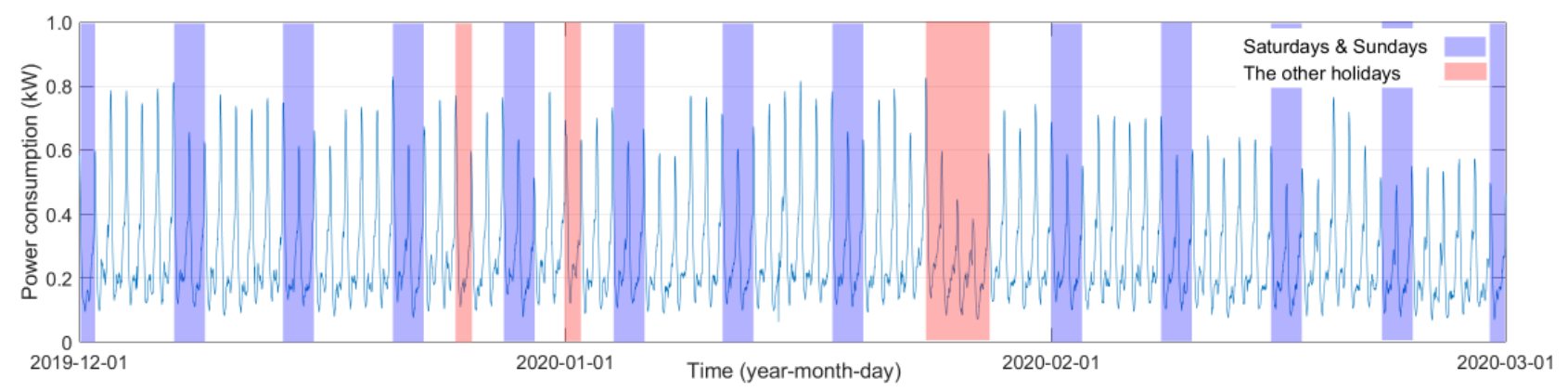

(a)

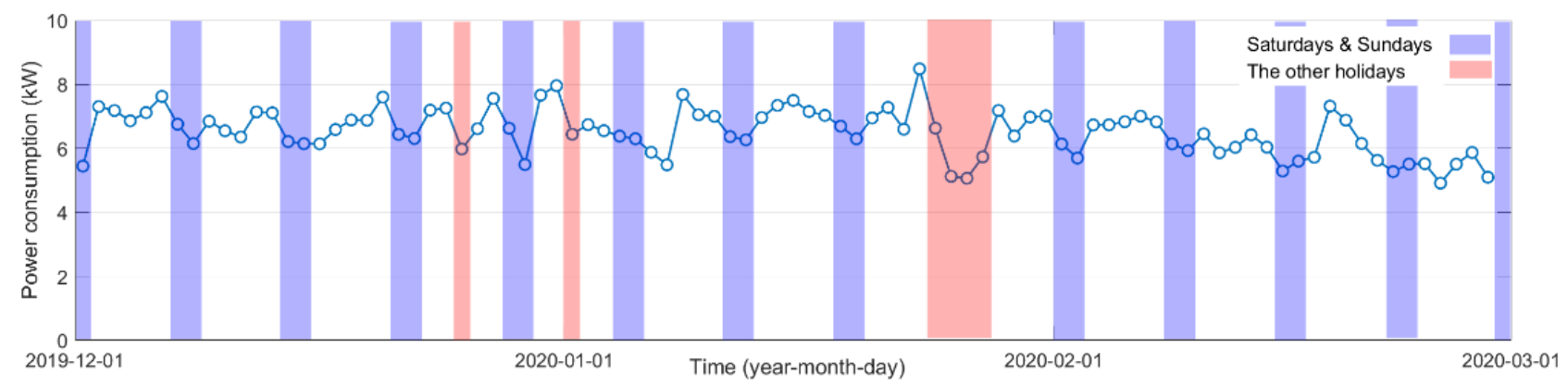

(b)

Figure 2. Periodicity analysis result of Electric Vehicle Charging (EVC) demand data: (a) 15-minutely average, (b) daily average.

\subsubsection{Variances}

Due to the daily periodicity, the overall demand pattern was confirmed with generally constant pattern as shown in Figure 3. In particular, the response to the time-of-use (TOU) tariff plan for EV charging, the demand was the highest after 23:00 when the off-peak pricing period began, and the demand and variance around 07:00 were the lowest. The result means that when dividing the data to analyze them in units of a daily cycle, splitting the data from 07:00 to 06:45 of the next day can preserve the load characteristics much 
more than from 00:00 to 23:45. Additionally, through confirming that there was a higher demand for EV charging between 18:30 and 03:30 on weekdays than on weekends and holidays, the reason of the aforementioned demand differences between weekdays and weekends/holidays could be identified.

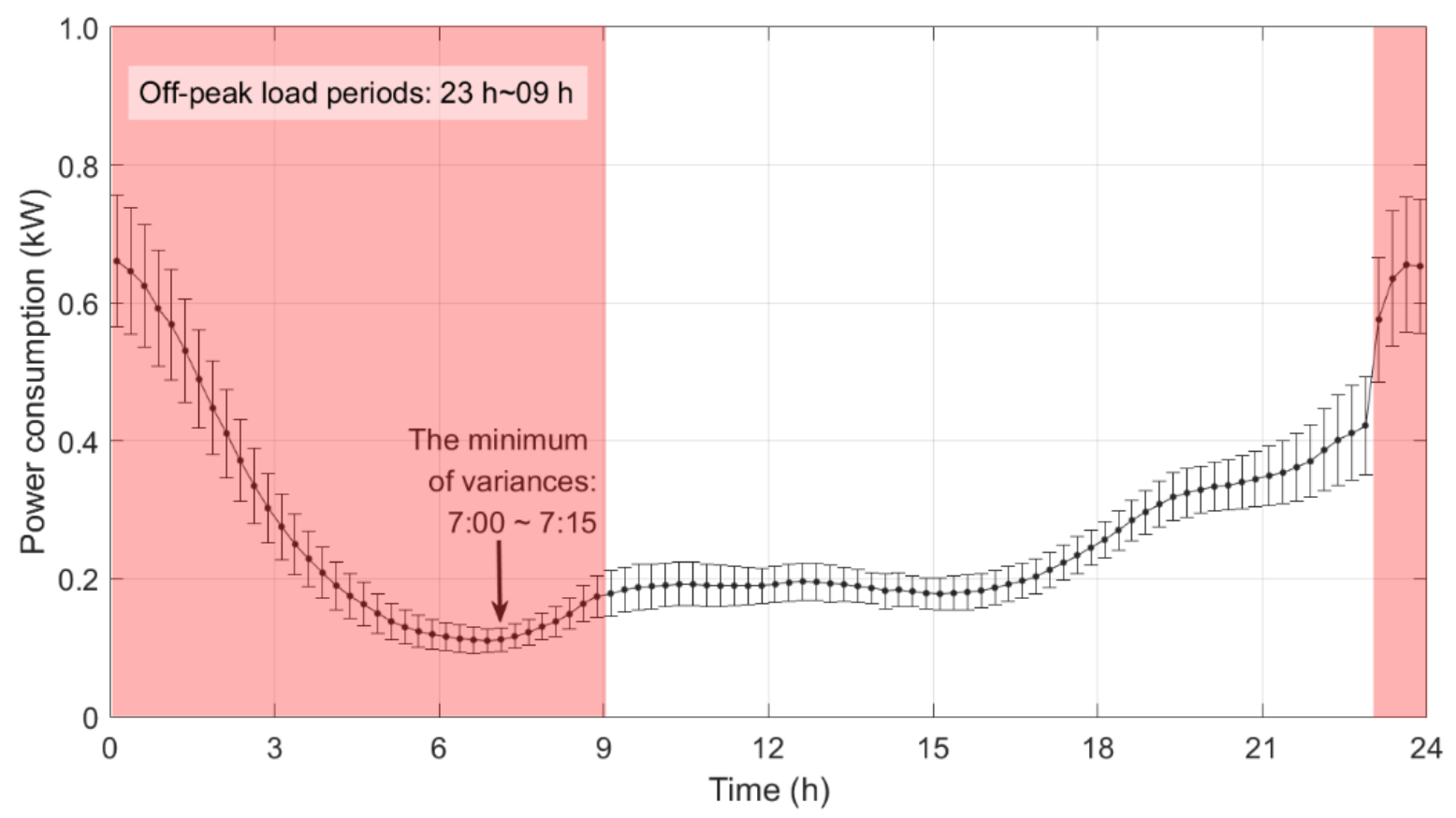

Figure 3. Variance analysis result of EVC charging demand data.

\subsubsection{EV User Segmentation}

To evaluate the demand flexibility based on charging characteristics of EV users, the representative charging profiles for clusters were created separately through customer segmentation. In this study, we used k-means++ data clustering methodology that has proven its performance in various research fields with the pre-determination of the number of clusters as five through Davies-Bouldin index evaluation [17]. According to the aforementioned periodicity characteristics, a total of three representative input profiles were proportionally applied to each weight in the dataset: a profile of working days (Mon-Fri) and two profiles of non-working days (weekends or holidays). The results of the segmentation into five groups according to the daily pattern by setting the standard time of the daily period to 07:00 are presented in Table 1 and Figure 4. The characteristics of the group pattern were classified into light-use, late-night charging, evening charging, morning charging and late-night heavy-use, respectively, and the light-use customers accounted for more than $50 \%$ of the total.

Table 1. EV user segmentation results.

\begin{tabular}{ccc}
\hline Group Number & Group Characteristics & Proportion \\
\hline 1 & Light-use & $57.1 \%$ \\
2 & Late-night charging & $17.6 \%$ \\
3 & Evening charging & $16.7 \%$ \\
4 & Morning charging & $4.8 \%$ \\
5 & Late-night heavy-use & $3.9 \%$ \\
\hline
\end{tabular}




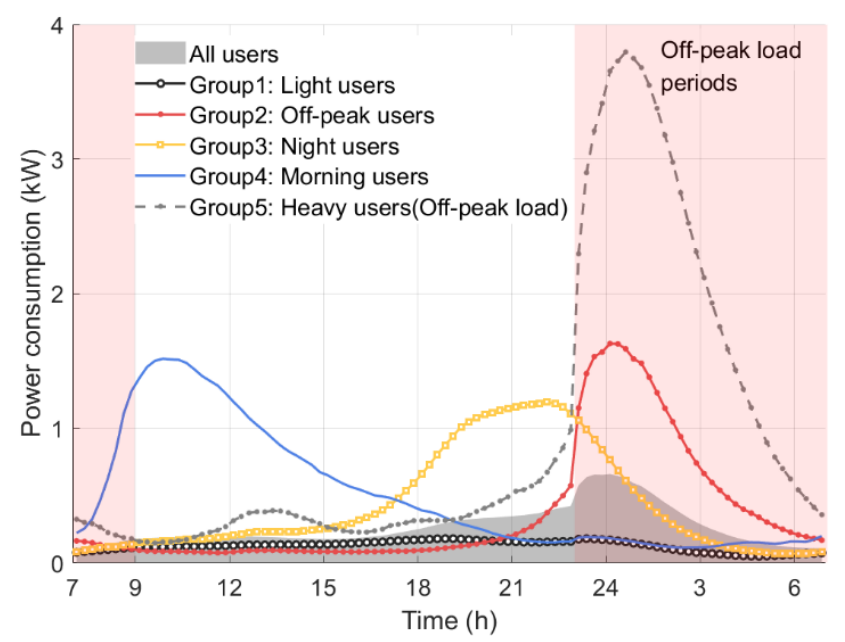

Figure 4. Representative demand profiles of EV user-segmented groups.

\section{Results and Discussions}

\section{Comparison of Flexibility Scores}

To estimate the demand flexibility score of EVC loads, the seasonal demand characteristic must be pre-reviewed, and the score was calculated based on the seasons and ramp-up/down intervals, respectively. In this study, the flexibility scores of group 1 to group 5 were evaluated and compared only for the winter season based on the data collected during the limited experimental period. According to the proposed scoring method in Section 2, the scores for entire groups averaged at approximately 0.0273 as shown in Table 2. According to the results, Group 4 was determined to have a demand flexibility because there was an opportunity to shift the morning charging pattern into the daytime, which is a ramp-up time period, and Group 3 was also a good FDR with the same reason as above.

Table 2. Flexibility score result (season: winter; resources: groups 1 to 5).

\begin{tabular}{|c|c|c|c|}
\hline Group & Score & $\begin{array}{c}\text { Case } 1 \\
\text { (Ramp-Up Interval) }\end{array}$ & $\begin{array}{c}\text { Case } 2 \\
\text { (Ramp-Down Interval) }\end{array}$ \\
\hline \multirow{4}{*}{ Group 1} & $F S$ & 0.456 & 0.435 \\
\hline & $C S$ & 0.503 & 0.554 \\
\hline & OS & 0.038 & 0.049 \\
\hline & $S$ & 0.009 & 0.012 \\
\hline \multirow{4}{*}{ Group 2} & FS & 0.498 & 0.494 \\
\hline & $C S$ & 0.502 & 0.565 \\
\hline & OS & 0.020 & 0.025 \\
\hline & $S$ & 0.005 & 0.007 \\
\hline \multirow{4}{*}{ Group 3} & $F S$ & 0.629 & 0.655 \\
\hline & $C S$ & 0.477 & 0.668 \\
\hline & OS & 0.064 & 0.186 \\
\hline & $S$ & 0.019 & 0.081 \\
\hline \multirow{4}{*}{ Group 4} & $F S$ & 0.685 & 0.508 \\
\hline & $C S$ & 0.631 & 0.544 \\
\hline & OS & 0.164 & 0.078 \\
\hline & $S$ & 0.071 & 0.021 \\
\hline \multirow{4}{*}{ Group 5} & $F S$ & 0.685 & 0.508 \\
\hline & $C S$ & 0.538 & 0.579 \\
\hline & OS & 0.067 & 0.077 \\
\hline & $S$ & 0.025 & 0.023 \\
\hline
\end{tabular}


The scores for Case 1 and 2 were averaged 0.0258 and 0.0288 , respectively. Accordingly, in the ramp-down interval, there was a tendency to receive higher scores for the FDR participation performance of customers than in the ramp-up interval. Considering the increasing load usage pattern among household EVC owners in the evening, quantitative indicators have identified more opportunities to reduce the load through EV charging schedule adjustments.

Additionally, in order to analyze the meaning of the scores, the flexibility evaluation result of EVC loads was compared with of industrial loads, which are suitable for FDR participation with a large and consistent power consumption pattern. For example, the scores for Case 1 and 2 of cement plants were averaged 0.2158 and 0.3173 with $61.04 \%$ of demand reduction capacity ratio [16].

The typical $O S$ of EVCs was significantly low and caused $S$ to be undervalued as a whole. Since the collection period of the dataset is only three months in winter and the total number of customers is small, the variance of the charging pattern of the segmented groups and the operation variability in each unit time interval were not considered in a heterogeneous environment. According to the authors' experiences, the more stable the power consumption was and the more even the cycle was, the closer the score could be to 1. This could be seen in resources with flexible range of temperature control (e.g., furnaces), or with clear periodicity of tasks. On the other hand, EV charging demands with binary characteristics are highly uncertain and difficult to get close to score of 1 . Compared to the industrial loads, the average difference in $S$ and $O S$ were 0.24 and 0.50, respectively [16].

Therefore, the clustering of customers can be an important role in high scores, which may be depend on the purpose of evaluating flexibility scores. Nevertheless, based on the data acquired from the perfectly same EVC type scattered throughout the residential sectors in the entire country, the high periodic similarity of the pattern was verified. In addition, this study presents meaningful implications considering that the scores can be used as the indicators to estimate demand flexibility for the day-ahead FDR operation plan in the short term and the network investment plan in the long term, respectively.

\section{Conclusions}

This paper proposed a demand flexibility evaluation model for segmented EVCs in the residential sector. The proposed methodology derives results with scores between 0 and 1, and it is designed such that the closer the score is to 1, the more likely it is to be worth as an FDR. The value of segmented EVC groups were quantified from the perspective of data analysis, reflecting the frequency of power consumption, the consistency of power consumption, and the operational capability. Prior to the demand flexibility evaluation, demand characteristic analysis was performed for typical EVC charging demand data with pre-processing. As a result, weekly and daily periodicity and variance were confirmed, and subsequently EV customers are segmented into five groups based on the similar charging demand patterns. The flexibility scores were calculated for the segmented groups and confirmed that the typical flexibility score during ramp-up and ramp-down intervals was 0.0273 .

The proposed method can be used as an indicator to determine whether an integrated EVC demand group is adequate to participate as a FDR. In addition, there is an implication to modify the proposed model according to the purpose of customer segmentation and scoring result analysis (e.g., FDR participation in various DR programs and time intervals with high reserve rate requirement, design for EV charging and discharging rates/incentives).

However, this study has certain limitations: a small dataset volume, the limited data collection period, and the lack of consideration for environment variability. Thus, to improve the reliability of the demand flexibility measurement, data collection and further studies are required based on heterogeneous environments such as rate plans, seasons, and $\mathrm{EV} / \mathrm{EVC}$ types. 
Author Contributions: Conceptualization, K.B.; Methodology Development and Simulation, K.B., S.K., and E.L.; Writing, Review, and Editing, K.B., E.L., Y.C., and J.K.; Project Management and Supervision, J.K. All authors have read and agreed to the published version of the manuscript.

Funding: This work was supported by the Korea Institute of Energy Technology Evaluation and Planning(KETEP) and the Ministry of Trade, Industry \& Energy(MOTIE) of the Republic of Korea (No. 20181210301380 and No. 20202010600010).

Institutional Review Board Statement: Not applicable.

Informed Consent Statement: Not applicable.

Data Availability Statement: Not applicable.

Conflicts of Interest: The authors declare no conflict of interest.

\section{Nomenclature}

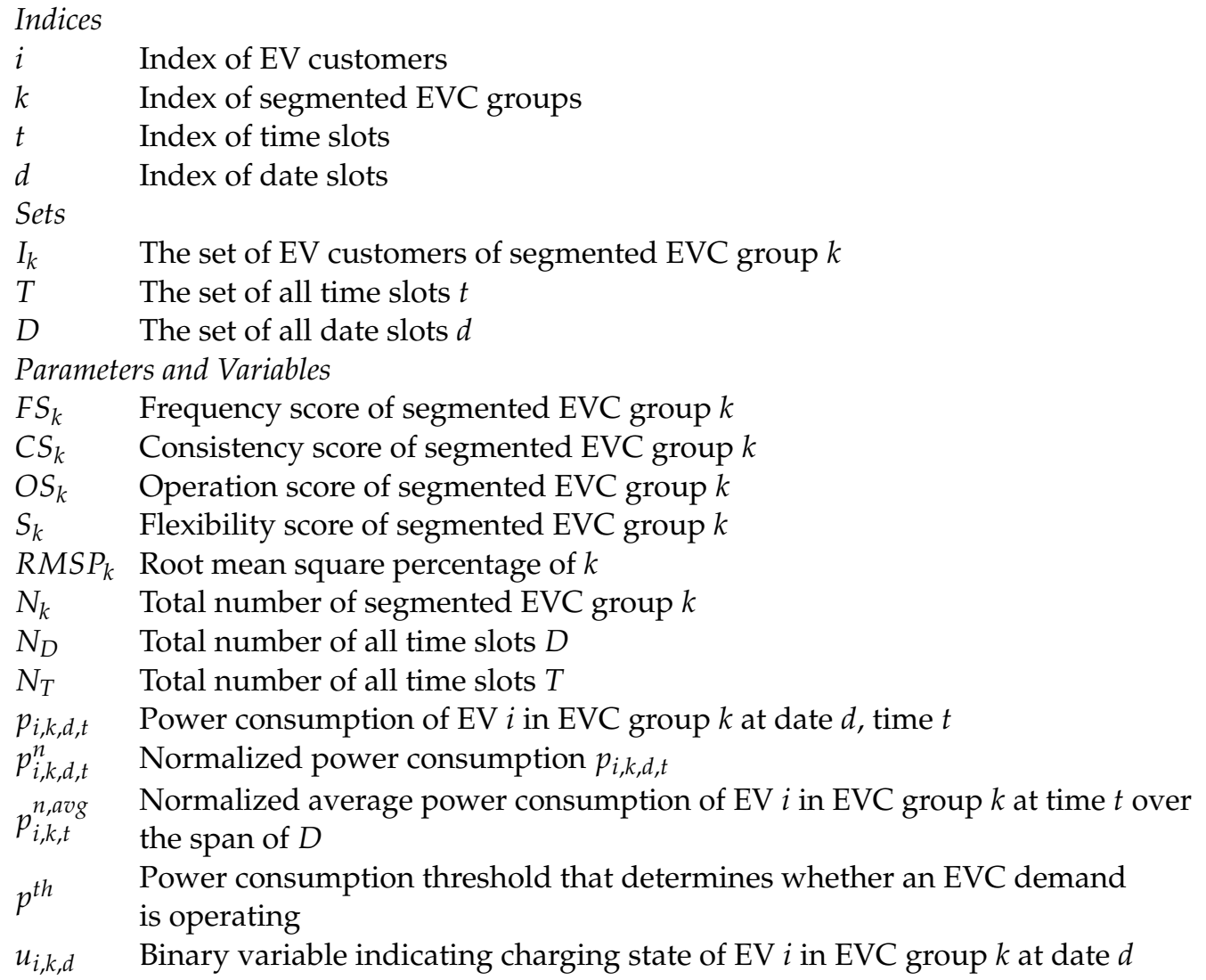

\section{References}

1. Tanaka, M. Real-time pricing with ramping costs: A new approach to managing a steep change in electricity demand. Energy Policy 2006, 34, 3634-3643. [CrossRef]

2. Strbac, G. Demand side management: Benefits and challenges. Energy Policy 2008, 36, 4419-4426. [CrossRef]

3. Heggarty, T.; Bourmaud, J.Y.; Girard, R.; Kariniotakis, G. Quantifying power system flexibility provision. Appl. Energy 2020, 279, 115852. [CrossRef]

4. Van Greet, E. Dealing with uncertainty in system planning-has flexibility proved to be an adequate answer? CIGRE Electra 1993, 151.

5. Mohandes, B.; El Moursi, M.S.; Hatziargyriou, N.; El Khatib, S. A review of power system flexibility with high penetration of renewables. IEEE Trans. Power Syst. 2019, 34, 3140-3155. [CrossRef]

6. Babatunde, O.M.; Munda, J.L.; Hamam, Y. Power system flexibility: A review. Energy Rep. 2020, 6, 101-106. [CrossRef]

7. Huber, M.; Dimkova, D.; Hamacher, T. Integration of wind and solar power in Europe: Assessment of flexibility requirements. Energy 2014, 69, 236-246. [CrossRef]

8. Lizana, J.; Friedrich, D.; Renaldi, R.; Chacartegui, R. Energy flexible building through smart demand-side management and latent heat storage. Appl. Energy 2018, 230, 471-485. [CrossRef] 
9. Ota, Y.; Taniguchi, H.; Nakajima, T.; Liyanage, K.M.; Baba, J.; Yokoyama, A. Autonomous distributed V2G (vehicle-to-grid) considering charging request and battery condition. In Proceedings of the 2010 IEEE PES Innovative Smart Grid Technologies Conference Europe (ISGT Europe), Gothenberg, Sweden, 11-13 August 2010; pp. 1-6.

10. Donadee, J.; Shaw, R.; Garnett, O.; Cutter, E.; Min, L. Potential benefits of vehicle-to-grid technology in California: High value for capabilities beyond one-way managed charging. IEEE Electrif. Mag. 2019, 7, 40-45. [CrossRef]

11. Schuller, A.; Flath, C.M.; Gottwalt, S. Quantifying load flexibility of electric vehicles for renewable energy integration. Appl. Energy 2015, 151, 335-344. [CrossRef]

12. Wu, Q.; Nielsen, A.H.; Østergaard, J.; Cha, S.T.; Marra, F.; Chen, Y.; Træholt, C. Driving pattern analysis for electric vehicle (EV) grid integration study. In Proceedings of the 2010 IEEE PES Innovative Smart Grid Technologies Conference Europe (ISGT Europe), Gothenberg, Sweden, 11-13 August 2010; pp. 1-6.

13. Corchero, C.; Gonzalez-Villafranca, S.; Sanmarti, M. European electric vehicle fleet: Driving and charging data analysis. In Proceedings of the 2014 IEEE International Electric Vehicle Conference (IEVC), Florence, Italy, 17-19 December 2014; pp. 1-6.

14. Sadeghianpourhamami, N.; Refa, N.; Strobbe, M.; Develder, C. Quantitive analysis of electric vehicle flexibility: A data-driven approach. Int. J. Elec. Power Energy Syst. 2018, 95, 451-462. [CrossRef]

15. Sajjad, I.A.; Chicco, G.; Napoli, R. Definitions of demand flexibility for aggregate residential loads. IEEE Trans. Smart Grid 2016, 7, 2633-2643. [CrossRef]

16. Lee, E.; Baek, K.; Kim, J. Evaluation of demand response potential flexibility in the Industry based on a data-driven approach. Energies 2020, 13, 6355. [CrossRef]

17. Aggarwal, C.C.; Reddy, C.K. Data Clustering: Algorithms and Applications; CRC Press: Boca Raton, FL, USA, 2016; pp. 87-110. 